\title{
A tandem-based compact dual-energy gamma generator*
}

\author{
A. Persaud, J.W. Kwan, M. Leitner, K.-N. Leung, B. Ludewigt, N. Tanaka, ${ }^{\dagger}$ W. Waldron, and S. Wilde \\ E.O. Lawrence Berkeley National Laboratory \\ 1 Cyclotron Road, Berkeley, California 94720, USA
}

\author{
A.J. Antolak, D.H. Morse, and T. Raber \\ Sandia National Laboratories, \\ Livermore, California, 94550, USA
}

\begin{abstract}
A dual-energy tandem-type gamma generator has been developed at E.O. Lawrence Berkeley National Laboratory and Sandia National Laboratories. The tandem accelerator geometry allows higher energy nuclear reactions to be reached, thereby alowing more flexible generation of $\mathrm{MeV}$ energy gammas for active interrogation applications. Both pasitively charged ions and atoms of hydrogen are created from negative ions via a gas stripper. In this paper, we show first results of the working tandem-based gamma generator and that a gas stripper can be utilized in a compact source design. Preliminary results of monoenergetic gamma production are shown.
\end{abstract}

\section{INTRODUCTION}

The detection of special nuclear material (SNM) can be achieved in active interrogation via neutrons or gamma rays. Neutrons have the advantage that they can create fission at low (thermal neutron) energies. However, they can be shielded by low-Z material. Gamma rays, on the other hand, have good penetration for low- $Z$ material, but higher energies are needed to penetrate dense materials and to cause photofission in SNM, since photofission cross-sections have a threshold around $5.5 \mathrm{MeV}$ and peak around $14 \mathrm{MeV}[1]$. One approach to create gammas in this energy range is to utilize low-energy nuclear reactions. The advantage of nuclear reactions is that monoenergetic $\mathrm{MeV}$-energy gammas can be created reducing the overall dose applied during interrogation. Furthermore, nuclear reactions allow the use of incident particle energies that are smaller by a factor of 20 compared to Bremsstrahlung sources. This translates into smaller accelerator structures, which makes the gamma source compact and aiso reduces the overall system cost.

In the present system, two nuclear reactions are investigated for the generation of gammas in a compact setup: ${ }^{11} \mathrm{~B}(p, \gamma){ }^{12} \mathrm{C}$ and ${ }^{19} \mathrm{~F}(\mathrm{p}, \gamma \alpha){ }^{16} \mathrm{O}$. The first reaction has a production cross-section resonance at a proton energy of $163 \mathrm{keV}$ and produces monoenergetic gammas at energies of $4.4,11.7$ (97\% emission fraction) and $16.1 \mathrm{MeV}$ ( $3 \%$ emission fraction)[1]. The maximum cross-section for this sharp resonance is $0.16 \mathrm{mb}$. The fluorine-based reaction has a resonance with a much higher cross-section

* Contributed paper, published as part of the Proceedings of the 13th Internationa! Conference on Ion Sources, Gatlinburg, Tennessee, September 2009.

${ }^{\dagger}$ Author to whom correspondence should be addressed. Electronic mail: aparsaudolbl.gov

$\ddagger$ Present address: Tohoku University, Sendai 980-77, Japan of $160 \mathrm{mb}$ at roughly twice the energy of the boron reaction $(340 \mathrm{keV})$, but the created gammas have an energy of $6.1 \mathrm{MeV}$ (Ref. [1]), which is on the lower end of the SNM photofission cross-section (see Ref. [2] for more information on this topic).

To achieve the required acceleration voltages in a compact design, a tandem accelerator configuration was chosen to reduce the maximum voltage needed in the device. This paper addresses the design and layout of the tandem-type gamma generator as well as the negative ion source and computer simulations for the beam transport. First resuits from beam-on-target runs and gamma spectra from the operational device are presented. At the end of the paper, possible improvements and the limits of this approach are discussed.

\section{TANDEM GAMMA GENERATOR}

The overall size (footprint) of the gamma generator system is primarily driven by the high voltage needed for the nuclear reactions. By using low-energy nuclear reaction resonances and by using a tandem-type accelerator structure (see Fig. 1), the few hundred kilovolts high voltage needed to produce these protons can be cut by factor of two. For example, a voltage of $180 \mathrm{kV}$ at the high voltage terminal in the tandem generator will provide $360 \mathrm{keV}$ protons for the $\mathrm{p}^{-19} \mathrm{~F}$ reaction to occur at its $340 \mathrm{keV}$ resonance. Since lower voltages are used, another advantage is that a a tandem generator can be designed with only one insulator column (instead of two) by relying on vacuum to provide the needed insulation.

\section{A. Ion source and extraction system}

A negative hydrogen ion source is being used based on a design that was originally developed at E.O. Lawrence Berkeley National Laboratory for the Spallation Neutron 


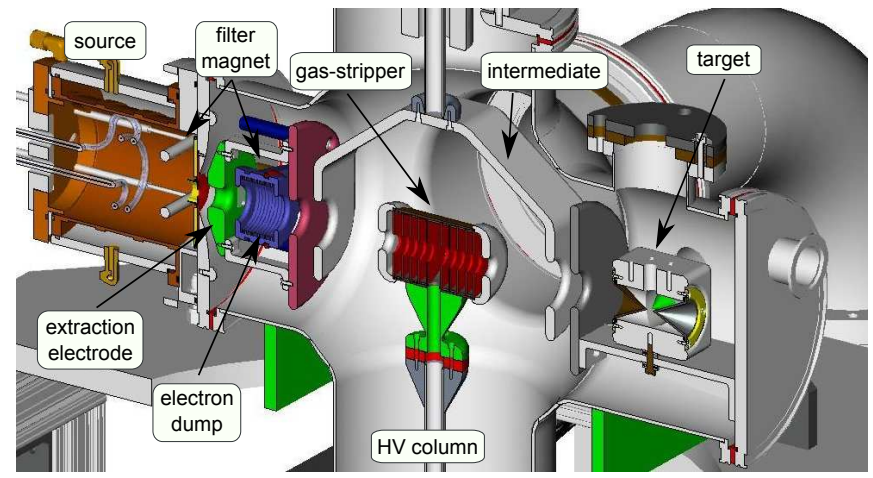

FIG. 1: (Color online) A CAD-drawing of the devise, showing all beamline components.

Source (SNS) project. It consists of a rf-driven multicusp source with integrated filter magnets $(150 \mathrm{G}$ on axis) [3]. The extraction region features a cesium collar where cesium can be dispensed to increase surface conversion of negative ions. The negative ions are extracted via a $30 \mathrm{kV}$ extraction electrode, which is followed by a $5 \mathrm{kV}$ electron-dump and another $30 \mathrm{kV}$ electrode. These three electrodes form an einzel lens structure to focus the ion beam. At the same time a magnetic filter is used in this region to deflect coextracted electrons into the electrondump. A deacceleration einzel lens is used to dump the electrons at lower potential. The deflection of the ions due to the magnetic field could be neglected since the beam spot size and emittance are not of a concern in this system (a large beam spot at the target is beneficial to distribute the beam load on the target).

\section{B. Gas stripper}

The einzel lens system is followed by an intermediate electrode at a potential of $70 \mathrm{kV}$ to screen the gasstripper, which is sitting at the center of the system at a high voltage (up to $185 \mathrm{kV}$ ). Here the negative hydrogen ions interact with an argon gas load, are stripped of their electrons, and are reaccelerated to a target at ground potential. The stripper chamber has a length of $11 \mathrm{~cm}$ and includes several apertures to reduce the gas flow into the main chamber. Limiting the gas flow into the chamber was initially a concern since the compactness of the design leads to higher electric fields and therefore easier break down, especially at higher pressures in parts of the chamber. To prevent such breakdown, the system was designed for a maximum field gradient of $25 \mathrm{kV} / \mathrm{cm}$. The argon gas is fed into the system through the oil tank and the high voltage column. A remote-controlled needle valve has been added inside the oil tank to enable the control of the argon flow.

\section{Nuclear reaction target}

The target assembly consists of a water-cooled cubeshaped holder where four different gamma production targets can be mounted. The targets themselves are cone-shaped copper pieces, to provide good cooling characteristics, which are coated with boron or lithium fluoride on their inside surface. The target diameter is $2.5 \mathrm{~cm}$, roughly the same size as the calculated beam spot.

Alternatively, a Faraday cup with a similar opening aperture and an internal secondary electron suppression electrode can be mounted at the target position. In this case, the beam current is collected from an isolated backplate behind the suppressor.

\section{Chamber}

The prototype tandem generator has been built using a small six-way stainless steel "cross" housing as shown in Fig. 1. The ports are used for the ion source, the target, a $200 \mathrm{kV}$ high voltage column, a $100 \mathrm{kV}$ feed-through, a flange with low voltage electrical feed-throughs, and water cooling feed-throughs for the magnet assembly, and a 1000 1/s turbomolecular pump. The chamber was custom made with a mirror finish and rounded corners, machined to provide better high voltage holding characteristics. The high voltage column needed to be encapsulated on the air side in an high dielectric material provided by an oil tank. A $200 \mathrm{kV}, 4 \mathrm{~mA}$ power supply was used for the experiments described below. The complete length of the system, including ion source and target, is roughly $65 \mathrm{~cm}$. The total footprint including the power supply and the oil tank is about $2 \mathrm{~m}^{2}$.

\section{SIMULATIONS}

The design of the system was aided by computer simulations of the ion beam transport using the Warp3D computer program[4]. The model included magnetic fields, charge exchange defined by a specified pressure profile, and treated the effects of space charge and secondary electrons correctly. The simulations were used to design elements for maximizing beam-on-target, minimizing local electric field gradients, and optimizing the performance of the magnetic filters to dump coextracted electrons. Confining the secondary electrons in the einzel lens region required incorporating additional magnetic filters in the system. Nevertheless, it was later found that electrons were still able to make it through the extraction system, so further improvements in the electron suppression system are possible. The results of the simulated ion optics calculations can be seen in Fig. 2. 


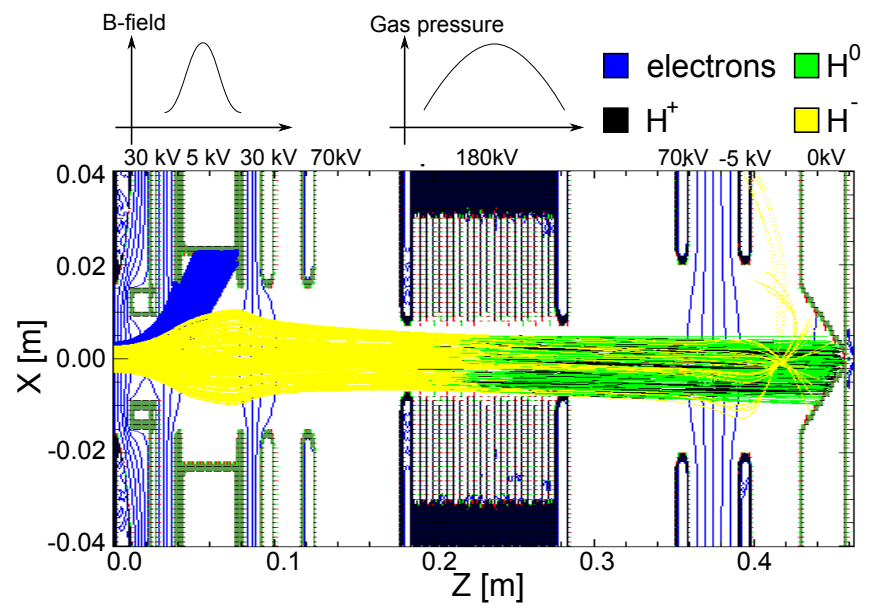

FIG. 2: (Color online) Computer simulation of the device. The magnetic field and the gas pressure profile are shown on the top as well as the applied voltages.

\section{EXPERIMENTS \& RESULTS}

High-potting experiments both with and without a gas load of up to $6.7 \times 10^{-2} \mathrm{~Pa}\left(5 \times 10^{-4}\right.$ Torr $)$ in the main chamber were successful. The highest voltage the system was operated at was $185 \mathrm{kV}$, and reaching $170 \mathrm{kV}$ for the $\mathrm{p}-{ }^{19} \mathrm{~F}$ reaction is routinely possible. The main source of the gas load is generated by argon from the gas stripper. Using only the source at normal operation pressure of $2.7 \mathrm{~Pa}\left(2 \times 10^{-2}\right.$ Torr $)$ in the source body leads to a pressure in the chamber of only $1.1 \times 10^{-2} \mathrm{~Pa}\left(8 \times 10^{-5}\right.$ Torr $)$. The pressure in the source chamber was measured with a Baracell with the source off because of rf interference effects with the readout electronic, while the chamber pressure was measured via an ion gauge.

For $\mathrm{H}^{-}$extraction, the $2 \mathrm{MHz}$ rf source is operated in pulsed mode with a pulse length of $100 \mu$ s and a repetition rate of $20-100 \mathrm{~Hz}$. The forward power is normally run at a level of $10 \mathrm{~kW}$. A Faraday cup was used for extraction tests and a total ion current of about $1 \mathrm{~mA}$ was measured. Figure 3 shows the pressure dependence of the Faraday cup current. These measurements use a $-400 \mathrm{~V}$ suppression bias on the Faraday cup.

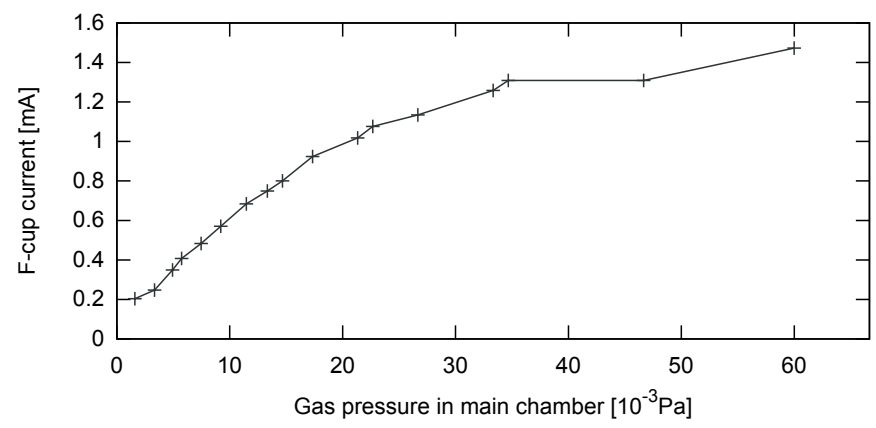

FIG. 3: The argon pressure in the gas stripper had a strong effect on the ion current (data taken at $100 \mathrm{kV}$ ).
Initial experiments to generate gammas used a solid boron target mounted in the target holder. A $7.6 \mathrm{~cm}$ diameter $\mathrm{NaI}$ detector was used to collect the gamma ray spectra at a distance of $50 \mathrm{~cm}$ from the target. The spectrum shown in Fig. 4 was acquired in 12 min, giving a

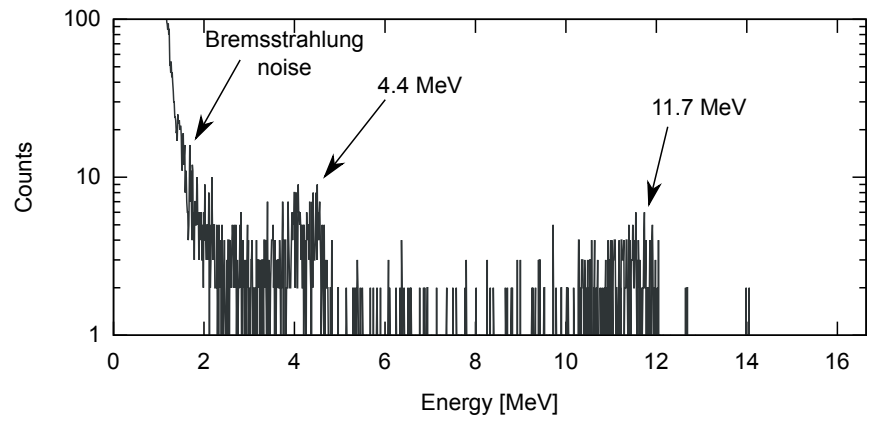

FIG. 4: Gamma spectrum of a boron target at $100 \mathrm{kV}, 1 \%$ duty cycle, $8 \mathrm{~kW}$ rf power.

gamma count rate of about 1 count/s at the detector $(1 \%$ duty cycle), consistent with results from earlier experiments with a single-ended setup[5]. The gamma lines at 4.4 and $11.7 \mathrm{MeV}$ are clearly visible. The acceleration voltage for these experiments was $100 \mathrm{keV}$. Figure 5 shows a spectrum from a lithium fluoride target taken at an acceleration voltage of $174 \mathrm{keV}$. Here, the ${ }^{19}{ }^{19} \mathrm{~F}$ gamma peak at $6.1 \mathrm{MeV}$ can be clearly identified. The fluorine data were taken at a duty cycle of $0.2 \%$ resulting in a count rate of 3 counts $/ \mathrm{s}$. A higher count rate in the p- ${ }^{11} \mathrm{~B}$ reaction was expected but not observed, possibly due to a lower beam current and a not optimal matched beam energy.

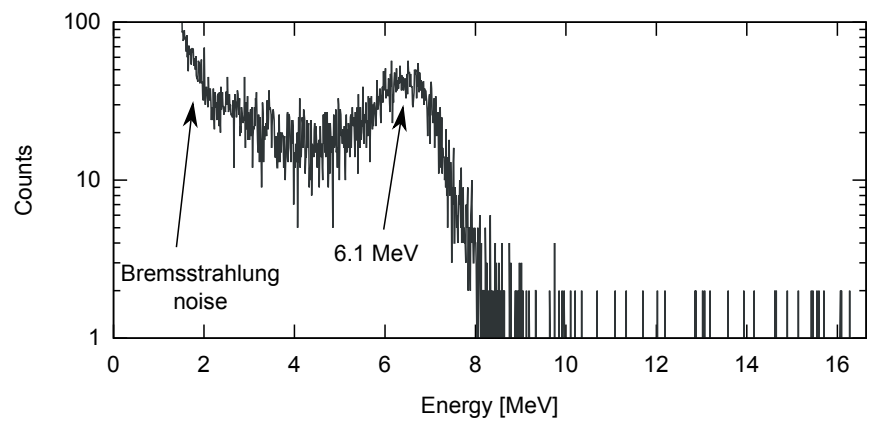

FIG. 5: Gamma spectrum of a lithium fluoride target at $174 \mathrm{kV}, 0.2 \%$ duty cycle, $5 \mathrm{~kW}$ rf power

Initial experiments revealed insufficient suppression of secondary electrons, which led to the production of intense Bremsstrahlung radiation at the gas cell. The Bremsstrahlung created unwanted noise in the detector and also caused heating of beam elements. 


\section{OUTLOOK}

Preliminary experimental measurements of nuclear reaction-produced gamma rays are presented from a prototype tandem-type monoenergetic photon generator. The experiments indicate that several improvements can be made to the system to produce higher beam current and, consequently, higher gamma yield. For example, although the capability exists, no experiments have been performed using cesium to increase negative ion production similar to the $40 \mathrm{~mA}$ currently achieved by the SNS ion source[6]. The tandem generator was designed to be able to operate with currents up to $50 \mathrm{~mA}$, which would lead to significantly increased gamma production. By further increasing the duty cycle to $10 \%$, gamma count rates of $4 \times 10^{5}$ counts/s should be achievable in $\mathrm{p}-{ }^{19} \mathrm{~F}$ for improved SNM detection via active interrogation (this would be equivalent to a total gamma yield of $3 \times 10^{8}$ counts/s into $\left.4 \pi\right)$.

The footprint of the system could also be reduced by integrating the high voltage feed-through and power supply. The generator itself can be made more compact by implementing a solid foil stripper to reduce the ion source-to-target distance and eliminating the associated pumping requirements.

\section{CONCLUSIONS}

We have demonstrated the operation of a compact tandem accelerator for gamma production that uses a gas charge exchange cell and a single dc high voltage column. We show first results for creating nuclear reaction-based gammas using boron and fluorine targets. Such a compact monoenergetic gamma generators is well-suited for SNM detection and other applications that benefit from a small footprint, for example, accelerator mass spectrometry or medical applications, for which the ion source part would have to be re-engineered to produce good beam emittance. Furthermore this approach can be used to replace radioactive isotope sources.

\section{Acknowledgments}

The authors would like to thank Daniel Buller and Paula Provencio at Sandia National Laboratories for coating the copper targets. This work was performed under the auspices of the US Department of Energy, NNSA Office of Nonproliferation Research and Engineering (NA-22) by E.O. Lawrence Berkeley National Laboratory under Contract DE-AC02-05CH11231 and by Sandia National Laboratories under contract DE-AC0494AL85000.
[1] J. R. Tesmer and M. A. Nastasi, eds., Handbook of modern ion beam materials analysis (Materials Research Society, 1995).

[2] A. Antolak, B. Doyle, M. King, F. McDaniel, D. Morse, P. Provencio, T. Raber, and P. Rossi, Sandia National Laboratories Report SAND2008-7934 (2008), URL http://infoserve.sandia.gov/sand_doc/ 2008/087934.pdf.

[3] R. F. Welton, M. P. Stockli, S. N. Murray, and R. Keller, Rev. Sci. Instrum. 75, 1793 (2004).

[4] A. Friedman, D. Grote, and I. Haber, Phys. Fluids B 4
2203 (1992).

[5] M. J. King, A. J. Antolak, K.-N. Leung, D. H. Morse, T. N. Raber, and B. L. Doyle, AIP Conference Proceedings 1099, 619 (2009), URL http://link . aip.org/link/ ?APC/1099/619/1.

[6] R. F. Welton, M. P. Stockli, S. N. Murray, T. R. Pennisi, B. Han, Y. Kang, R. H. Goulding, D. W. Crisp, D. O. Sparks, N. P. Luciano, J. R. Carmichael, J. Carr, Rev. Sci. Instrum. 79, 02C721 (2008). 
This document was prepared as an account of work sponsored by the United States Government. While this document is believed to contain correct information, neither the United States Government nor any agency thereof, nor The Regents of the University of California, nor any of their employees, makes any warranty, express or implied, or assumes any legal responsibility for the accuracy, completeness, or usefulness of any information, apparatus, product, or process disclosed, or represents that its use would not infringe privately owned rights. Reference herein to any specific commercial product, process, or service by its trade name, trademark, manufacturer, or otherwise, does not necessarily constitute or imply its endorsement, recommendation, or favoring by the United States Government or any agency thereof, or The Regents of the University of California. The views and opinions of authors expressed herein do not necessarily state or reflect those of the United States Government or any agency thereof or The Regents of the University of California. 